\title{
A commentary on "Microablative radiofrequency versus pelvic floor muscle training for stress urinary incontinence: a randomized controlled trial"
}

\author{
Ghazaleh Rostaminia ${ }^{1}$ (D) \\ Received: 12 April 2021 / Accepted: 26 April 2021 / Published online: 20 May 2021 \\ (C) The International Urogynecological Association 2021
}

This prospective three-arm randomized clinical trial aimed to compare the effect of fractional microablative radiofrequency (RF) and pelvic floor muscle training (PFMT) against the combination of both therapies (RF + PFMT) for treating stress urinary incontinence (SUI) and genitourinary syndrome (GSM). One hundred seventeen post-menopausal women with stress dominant urinary incontinence in the absence of stage III or IV vaginal prolapse were recruited and allocated into three intervention groups ( $n=39$ in each group): group 1: 3 monthly sessions of RF; group 2: 12 PFMT weekly sessions; group 3: RF + PFMT simultaneously. Assessments at baseline and 30 days after the end of therapy were conducted using validated questionnaires and scales for urinary, vaginal and sexual functions and cytology for vaginal atrophy.

The mean age was $54.7 \pm 6.3$ years old. Fifteen women (12.8\%) failed to comply with the study protocol, and four participants were lost to follow-up. All groups were comparable in terms of demographic characteristics with no significant differences. SUI symptoms assessed by ICIQ-SF scores improved significantly in all three groups post-treatment $(p<0.001)$. However, the improvement was significantly greater in the RF + PFMT compared to the RF and PFMT groups $(p=0.002)$. There was evidence that all three therapies improved vaginal symptoms, according to ICIQ-VS total scores $(p<0.001)$. The RF group showed a more significant decrease in the symptom score ( -9.3 points) compared to the $\mathrm{RF}+$ PFMT $(-4.4)$ and PFMT $(-3.4)$ groups $(p=0.007)$. According to answers obtained from Question 4 of the ICIQ-VS, vaginal laxity improved after all treatments $(p<0.001)$, with no significant differences being observed between them $(p=0.323)$. The sensation of vaginal dryness was evaluated according to Question 7 of the ICIQ-VS questionnaire and showed improvement after RF $(p<0.001)$ and $\mathrm{RF}+$ PFMT $(p=0.024)$. Compared to other therapies, RF therapy was associated with the greatest shifts in sensation of vaginal dryness $(p=0.009)$. Ninety-two percent of participants reported feeling that they had been cured or that their symptoms had improved. More participants reported feeling that they had been cured in the RF and RF + PFMT groups than in the PFMT group $(p=0.032)$.

In summary, this study showed that women with SUI experienced objective and subjective improvement of UI with RF therapy - whether it was associated with PFMT or not - as well as with PFMT alone. Improvement of vaginal symptoms and dryness was greatest in the RF treatment, and vaginal laxity showed similar improvement in all three groups. While recent technological innovations including fractional laser and microablative radiofrequency are offering nonsurgical non-hormonal treatment for bundle symptoms of vaginal dryness, SUI, and vaginal laxity, the FDA has not yet determined whether it is safe or practical to use energybased devices for treating these conditions. It is indisputable that despite promising availability of short-term data, more robust, sham-controlled studies and knowledge of longer term outcomes are still needed.

Publisher's note Springer Nature remains neutral with regard to jurisdictional claims in published maps and institutional affiliations.
Ghazaleh Rostaminia

ghazalerostaminia@yahoo.com

1 Northshore University Health System, Skokie IL USA 